\title{
Inequalities that test locality in quantum mechanics
}

\author{
Dennis Dieks* \\ Institute for the History and Foundations of Science, Utrecht University, P.O. Box 80.000, 3508 TA Utrecht, The Netherlands
}

(Received 1 July 2002; published 6 December 2002)

\begin{abstract}
Quantum theory violates Bell's inequality, but not to the maximum extent that is logically possible. We derive inequalities (generalizations of Cirel'son's inequality) that quantify the upper bound of the violation, both for the standard formalism and the formalism of generalized observables (POVMs). These inequalities are quantum analogues of Bell inequalities, and they can be used to test the quantum version of locality. We discuss the nature of this kind of locality. We also go into the relation of our results to an argument by Popescu and Rohrlich [Found Phys. 24, 379 (1994)] that there is no general connection between the existence of Cirel'son's bound and locality.
\end{abstract}

DOI: 10.1103/PhysRevA.66.062104

\section{INTRODUCTION}

The violation of Bell's inequality by the predictions of quantum theory (both in its non-relativistic and relativistic versions) shows that quantum theory is non-local in the sense that its results cannot be reproduced by a hidden-variables theory in which measurement results depend only on the local settings of the measuring devices and on the properties of the objects being measured (a local hidden-variables theory). However, the maximum violation of Bell's inequality allowed by quantum theory is less than the maximum violation that is logically possible: quantum theory obeys Cirel'son's inequality. One might surmise that this is due to the fact that quantum theory does not abandon locality completely: after all, in situations of the Einstein-PodolskyRosen (EPR) type the measurements performed on one wing do not influence expectation values on the other wing (the no-signaling theorem; in the relativistic context this is the feature of relativistic causality). Perhaps compliance with a no-signaling demand restricts the extent to which Bell's inequality can be violated, and perhaps inequalities like Cirel'son's can be regarded as a touchstone of this kind of locality (in the same way as Bell's inequality is a touchstone for locality in the classical sense).

In this paper we show that this hypothesis is right: the fact that quantum theory does not violate Bell's inequality to the maximum logically possible extent is due to features of locality that are built into the theory. We derive a set of inequalities, and a strongest inequality representing this whole set, that can be regarded as quantum versions of Bell's inequality. To make the analogy with Bell inequalities clear we will analyze how locality is implemented in quantum theory, and in what sense the quantum theoretical inequalities we derive are based on locality assumptions. We will discuss how this relates to a result of Popescu and Rohrlich [3] that at first sight seems to show that the existence of Cirel'son's bound is unconnected with locality issues.

\section{CIREL'SON'S INEQUALITY}

Consider a probability space in which there are four stochastic functions, $A, a, B, b$, each of which can take the

*Electronic address: dieks@ @hys.uu.nl
PACS number(s): 03.65.Ud, 03.65.Ta

values +1 or -1 . The quantity $A B+A b+a B-a b=A(B$ $+b)+a(B-b)$ can only be +2 or -2 , from which it follows that the absolute value of its expectation value is smaller than 2:

$$
|\langle A B+A b+a B-a b\rangle| \leqslant\langle|A B+a b+a B-a b|\rangle=2 .
$$

This is the form of Bell's inequality that we will consider. The inequality is respected by physical quantities in classical theories, as long as these quantities can be represented by (stochastic) functions on one state space, with a joint probability distribution-which is ordinarily the case. We will discuss the connection with locality in Sec. V.

In quantum mechanics physical magnitudes are not represented by stochastic functions on a phase space, but by Hermitian operators on a Hilbert space. Let us now use $A, a, B$, $b$ to denote such operators that have eigenvalues +1 and -1 , and let us consider a combination of them that is analogous to the combination of quantities in Bell's inequality: $A B$ $+A b+a B-a b$, where we assume that the operators occurring in a product commute. As was first shown by Cirel'son [1], the modulus of the quantum mechanical expectation value of this expression is bounded by $2 \sqrt{2}: \mid\langle A B+A b$ $+a B-a b\rangle \mid \leqslant 2 \sqrt{2}$ - the upper bound can be attained, as shown by the example of the singlet state. So Bell's inequality can be violated by quantum theory; but the quantum expectation value stays well below the logically possible upper bound of the expression $|\langle A B+A b+a B-a b\rangle|$, namely 4 .

Cirel'son's inequality can be proved elegantly by observing [2] that if $A^{2}=a^{2}=B^{2}=b^{2}=1$ and $[A, B]=[A, b]$ $=[a, B]=[a, b]=0$, then

$$
C^{2} \equiv(A B+A b+a B-a b)^{2}=41-[A, a][B, b] .
$$

It follows from this that

$$
\langle C\rangle^{2} \leqslant\left\langle C^{2}\right\rangle \leqslant\|C\|^{2} \leqslant 4+4\|A\|\|a\|\|B\|\|b\|=8,
$$

or

$$
|\langle C\rangle| \leqslant 2 \sqrt{2} .
$$

An alternative simple proof, which is analogous to the above proof of Bell's inequality (1) and similar to proofs of other inequalities that we will give in Secs. III and IV, goes as follows. 
For a normed state vector $|\psi\rangle$, put $A|\psi\rangle \equiv|A\rangle, B|\psi\rangle$ $\equiv|B\rangle, a|\psi\rangle \equiv|a\rangle$ and $b|\psi\rangle \equiv|b\rangle$. Each of these four vectors has a norm that is $\leqslant 1$. We now have

$$
\begin{aligned}
|\langle C\rangle| & =|\langle\psi|C| \psi\rangle| \\
& =|\langle A \mid B+b\rangle+\langle a \mid B-b\rangle| \\
& \leqslant \||B\rangle+|b\rangle\|+\||B\rangle-|b\rangle \mid \\
& \leqslant \sqrt{2(1+\operatorname{Re}\langle B \mid b\rangle)}+\sqrt{2(1-\operatorname{Re}\langle B \mid b\rangle)} \\
& \leqslant 2 \sqrt{2} .
\end{aligned}
$$

The difference between this derivation and the derivation of Bell's inequality is that for numbers $B$ and $b$ with norm $\leqslant 1$ we have $|B+b|+|B-b| \leqslant 2$, whereas for vectors with norm $\leqslant 1$ we find $\||B\rangle+|b\rangle\|+\||B\rangle-|b\rangle \| \leqslant 2 \sqrt{2}$. In the latter case the maximum is attained when $|B\rangle$ and $|b\rangle$ are perpendicular.

In the earlier derivation the essential premise was that the operators $A, a$ commute with $B, b$. At first sight, derivation (2) does not make use of this premise. This impression is deceptive, however. The operator products occurring in $C$ are Hermitian operators (and therefore representations of physical quantities) if and only if the operators that are multiplied commute, and this leads to exactly the same commutativity requirement as before. One physical consequence of this commutativity requirement is that the operators $A$ and $a$ are jointly measurable with the operators $B$ and $b$. Moreover, it follows from the commutativity that it does not make any difference for the expectation values of the operators $B$ or $b$ whether they are measured together with $A$ or $a$ (the nosignaling theorem). Within the framework of the orthodox measurement formalism comeasurability and causal independence (in the sense of no signaling) therefore go together: they both hold if and only if the commutativity requirement is satisfied. In this case Cirel'son's inequality also holds.

\section{GENERALIZED MEASUREMENTS}

Above we followed the orthodox point of view about the mathematical representation of physical quantities in quantum theory, namely that physical quantities are represented by Hermitian operators. Within this framework joint measurability is equivalent to commutativity (which in turn leads to the no-signaling theorem in the context of the EPR experiment). But there is a more general treatment of measurements in quantum theory, first developed by Ludwig [4] and Davies [5], in which physical quantities correspond not to single operators but to collections of positive operators $M_{i}$ on the Hilbert space, such that

$$
M_{i} \geqslant 0, \quad \sum_{i} M_{i}=1
$$

If the possible outcomes of a measurement of the considered quantity are $m_{i}$, the probabilities of obtaining these values in a state $\rho$ of the system are given by $\operatorname{Tr} \rho M_{i}$. The mapping $m_{i} \rightarrow M_{i}$ is a positive operator valued mapping (POVM), representative of the associated physical quantity $\mathcal{M}$.
Two physical quantities $\mathcal{A}$ and $\mathcal{B}$, represented by sets of positive operators $\left\{A_{i}\right\}$ and $\left\{B_{j}\right\}$, respectively, are jointly measurable if there is a third quantity $\mathcal{O}$, represented by $\left\{O_{k}\right\}$, such that

$$
A_{i}=\sum k \in K_{i} O_{k}, \quad B_{j}=\sum_{k \in K_{j}^{\prime}} O_{k}
$$

where $\left\{K_{i}\right\}$ and $\left\{K_{j}^{\prime}\right\}$ are two partitions of the index set through which $k$ runs.

If there is an $\mathcal{O}$ satisfying Eq. (3) we can measure it, and infer information about the outcomes and their probabilities of both $\mathcal{A}$ and $\mathcal{B}$ by grouping together the results according to the two partitions. An important feature of this formalism is that commutativity of the two generalized observables $\mathcal{A}$ and $\mathcal{B}$ (in the sense that $A_{i} B_{j}=B_{j} A_{i}$ for all $i, j$ ) is a sufficient but not a necessary condition for their joint measurability. If $\mathcal{A}$ and $\mathcal{B}$ commute, the products $A_{i} B_{j}$ are positive operators characterizing the joint measurement of $\mathcal{A}$ and $\mathcal{B}$. But in general a joint measurement need not correspond to product operators (see for a critical analysis of the significance of these results [6]).

So in the EPR situation we could imagine a joint measurement of two non-commuting generalized observables $\mathcal{A}$ and $\mathcal{B}$, each pertaining to a different wing of the experiment. In this case it would no longer be true that the mere requirement of compatibility leads to causal independence (no-signaling), the product form of the joint measurement operators, and the validity of Cirel'son's inequality.

However, Busch and Singh [7] have shown for the EPR situation, treated by means of the POVMs formalism, that if the possible values and their probabilities of the quantity measured at one wing are required to be independent of which quantity is measured at the other side, the operators representing the generalized observables at one wing must commute with those at the other. It follows that in this case the operators corresponding to the joint measurement take on the product form again. So within the generalized measurements framework commutativity and product form are consequences of locality, in the sense of the impossibility of signaling.

If the measurements in the EPR experiment are represented by generalized observables, and if locality in the sense of impossibility of signaling is assumed, Cirel'son's inequality can again be derived. To see this, consider one pair of the four pairs of observables, $\mathcal{A}$ and $\mathcal{B}$, say. Because of the no-signaling requirement, the corresponding positive operators $A_{i}$ and $B_{j}$ commute, and the joint measurement of $\mathcal{A}$ and $\mathcal{B}$ can be represented by four positive operators $A_{i} B_{j}$, with $i, j= \pm 1$. The expectation value of the outcomes of this joint measurement, in the pure state $|\psi\rangle$, becomes

$$
\begin{array}{r}
\left\langle\psi\left|\left(A_{1} B_{1}-A_{1} B_{-1}+A_{-1} B_{-1}-A_{-1} B_{1}\right)\right| \psi\right\rangle \\
=\left\langle\psi\left|\left(A_{1}-A_{-1}\right)\left(B_{1}-B_{-1}\right)\right| \psi\right\rangle=\langle A \mid B\rangle,
\end{array}
$$

where $|A\rangle \equiv\left(A_{1}-A_{-1}\right)|\psi\rangle$ and $|B\rangle \equiv\left(B_{1}-B_{-1}\right)|\psi\rangle$. So for the purpose of calculating expectation values the generalized observables $\mathcal{A}, \mathcal{B}, \widetilde{a}, \widetilde{b}$ can each be represented by a single 
Hermitian operator, namely $\left(A_{1}-A_{-1}\right),\left(B_{1}-B_{-1}\right),\left(a_{1}\right.$ $\left.-a_{-1}\right)$ and $\left(b_{1}-b_{-1}\right)$, respectively; the joint measurements are represented by the corresponding products. Compared to the case discussed in Sec. II, the differences are that the operators $A_{i}, B_{j}, \ldots$ need not be projection operators, and the squares of $\left(A_{1}-A_{-1}\right),\left(B_{1}-B_{-1}\right), \ldots$ need not be 1 . The second proof of the Cirel'son inequality given in Sec. II goes nevertheless through, because the operators $\left(A_{1}-A_{-1}\right)$, $\left(B_{1}-B_{-1}\right), \ldots$ all have norms $\leqslant 1$.

Indeed,

$$
\|\left(A_{1}-A_{-1}\right)|\psi\rangle\left\|^{2}=\right\| A_{1}|\psi\rangle\left\|^{2}+\right\| A_{-1}|\psi\rangle \|^{2}-2\left\langle A_{1} \psi \mid A_{-1} \psi\right\rangle,
$$

whereas

$$
\begin{aligned}
\|\left(A_{1}+A_{-1}\right)|\psi\rangle \|^{2} & =\| A_{1}|\psi\rangle\left\|^{2}+\right\| A_{-1}|\psi\rangle \|^{2}+2\left\langle A_{1} \psi \mid A_{-1} \psi\right\rangle \\
& =1
\end{aligned}
$$

so that

$$
\|\left(A_{1}-A_{-1}\right)|\psi\rangle \|^{2}=1-4\left\langle A_{1} \psi \mid A_{-1} \psi\right\rangle .
$$

Because $A_{-1}=1-A_{1},\left[A_{1}, A_{-1}\right]=0$ and the inner products in Eqs. (5), (6) and (7) are real. This inner product is also $\geqslant 0$ :

$$
\left\langle A_{1} \psi \mid A_{-1} \psi\right\rangle=\left\langle\psi\left|A_{1}\right| \psi\right\rangle-\left\langle\psi\left|A_{1}^{2}\right| \psi\right\rangle,
$$

which is $\geqslant 0$ because $A_{1}$ has norm $\leqslant 1$ and only has eigenvalues $\lambda_{i}$ with $0 \leqslant \lambda_{i} \leqslant 1$.

Now introduce vectors $|a\rangle,|b\rangle$ in the obvious way: $|a\rangle$ $\equiv\left(a_{1}-a_{-1}\right)|\psi\rangle,|b\rangle=\left(b_{1}-b_{-1}\right)|\psi\rangle$. It follows from the above that the vectors $|A\rangle,|B\rangle,|a\rangle,|b\rangle$ all have norms $\leqslant 1$, just as the vectors denoted by the same symbols in Sec. II. Repeating the proof (2), we therefore find

$$
|\langle\mathcal{A B}+\mathcal{A} \widetilde{b}+\widetilde{a} \mathcal{B}-\widetilde{a} \widetilde{b}\rangle|=|\langle A \mid B+b\rangle+\langle a \mid B-b\rangle| \leqslant 2 \sqrt{2} .
$$

This inequality holds in every pure state $|\psi\rangle$. Its validity in any mixed state $\rho$ follows immediately.

\section{THE STRONGEST INEQUALITY}

Cirel'son's inequality is not the only nor the strongest one that can be derived from the locality (no-signaling) and therefore commutativity requirement. Put $X \equiv\langle\mathcal{A} \widetilde{b}+\widetilde{a} \mathcal{B}\rangle$ and $Y \equiv\langle\mathcal{A B}-\widetilde{a} \widetilde{b}\rangle$. Cirel'son's inequality can now be written as

$$
|X+Y| \leqslant 2 \sqrt{2} .
$$

In the $X, Y$ "correlation plane" this inequality restricts the points $(X, Y)$ to the strip between the two lines

$$
X+Y= \pm 2 \sqrt{2} .
$$

But by a minimal change in the proof of Sec. II it immediately follows that also the following inequality holds:

$$
|X-Y| \leqslant 2 \sqrt{2}
$$

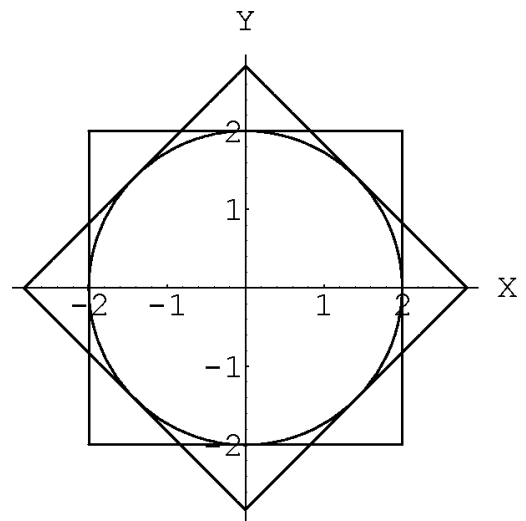

FIG. 1. The $X, Y$ plane. The slanted square represents inequalities (10) plus (12), the circle inequality (15).

so that the points must also lie in the strip bounded by the lines

$$
X-Y= \pm 2 \sqrt{2} .
$$

We also have the obvious inequalities $|X| \leqslant 2,|Y| \leqslant 2$, so that the allowed points $(X, Y)$ must be in the intersection of the interiors of the two squares indicated in Fig. 1. It further turns out that they must be inside (or on the sides of) all squares that result from these just-mentioned squares by applying an arbitrary rotation around an axis through the origin of the $X, Y$ plane and normal to this plane. To prove this, consider the expression $|X \sin \varphi+Y \cos \varphi|$. We have

$$
\begin{aligned}
&|X \sin \varphi+Y \cos \varphi| \\
&=|\langle A \mid B \cos \varphi+b \sin \varphi\rangle+\langle a \mid B \sin \varphi-b \cos \varphi\rangle| \\
& \leqslant\||B \cos \varphi\rangle+|b \sin \varphi\rangle|+\|| B \sin \varphi\rangle-|b \cos \varphi\rangle \| \\
& \leqslant \sqrt{\sin ^{2} \varphi+\cos ^{2} \varphi+2 \operatorname{Re}\langle B \mid b\rangle \sin \varphi \cos \varphi} \\
& \quad+\sqrt{\sin ^{2} \varphi+\cos ^{2} \varphi-2 \operatorname{Re}\langle B \mid b\rangle \sin \varphi \cos \varphi} \\
& \leqslant \sqrt{\sin ^{2} \varphi+\cos ^{2} \varphi}+\sqrt{\sin ^{2} \varphi+\cos ^{2} \varphi} \\
&= 2 .
\end{aligned}
$$

Cirel'son's inequality and the other inequalities mentioned earlier in this section are special cases of this general set of inequalities (in which $\varphi$ can take arbitrary values). It should be noted that these proofs apply both to the case of ordinary observables and to the case of generalized observables.

It is clear from the geometry of Fig. 1 that the requirement that all the inequalities (14) be satisfied leads to the inequality

$$
X^{2}+Y^{2}=\langle\mathcal{A} \widetilde{b}+\widetilde{a} \mathcal{B}\rangle^{2}+\langle\mathcal{A B}-\widetilde{a} \widetilde{b}\rangle^{2} \leqslant 4 .
$$

All points $X, Y$ are inside or on the circumference of a circle with radius 2 .

Inequality (15) (which was recently proved directly, by a variational argument, for the case of ordinary spin observables by Uffink [8]) summarizes all generalized Cirel'son inequalities (14). All values $X, Y$ that satisfy (15) also satisfy 
all Cirel'son inequalities (14); but satisfaction of a finite number of inequalities of Eq. (14) is not sufficient to guarantee satisfaction of Eq. (15). Moreover, each point on the circumference of the circle can actually be attained, because the bound of the corresponding generalized Cirel'son inequality can be attained (the one resulting in a line tangent to the circle in the point in question). Inequality (15) is therefore the strongest inequality in terms of $X, Y$ that follows from the requirement of commutativity.

\section{LOCALITY}

Bell's inequality (1) is valid for an arbitrary quadruple of stochastic functions on one probability space, and as such is not immediately connected with locality issues. The link with locality comes in via the application of Eq. (1) to situations of the Einstein-Podolsky-Rosen type in which $A$ and $a$, and $B$ and $b$, stand for measurements on the space-like separated wings 1 and 2 of the experiment, respectively. An experimenter at 1 can choose between measuring $A$ and $a$; her or his colleague at 2 has the choice between $B$ and $b$. The combined measurement on parts 1 and 2 is represented by the product of the individual single system result functions. This is justified by a locality assumption: a measurement of a physical quantity on one wing of the experiment has no influence at the other wing. On the basis of this assumption one-wing quantities are represented by one and the same function, regardless of whether, and if so which, measurement is performed at the other side. Both the possible measurement outcomes and their probabilities are insensitive to choices made at the other side.

An obvious silent assumption in this is that $A, a, B, b$ correspond to characteristic measurement devices and interactions. The device corresponding to $A$, e.g., should remain the same in different instances - that the possible outcomes and corresponding probabilities remain the same is by itself not enough. Consider to make this clear a Stern-Gerlach device at wing 1 that undergoes a rotation depending on the choice between $B$ and $b$ : although the possible outcomes would still be +1 and -1 and the probabilities would remain equal to $1 / 2$ (if the device measures the spin of a spin-1/2 particle), this would not constitute one specific measured quantity. Spin along different axes would be measured. In spite of the fact that all measurement results could be represented by the same function $A$, the rotation of the corresponding device would signal nonlocality. Within the quantum formalism such a noninvariance of the measuring procedure could easily lead to a violation of Eq. (15). An explicit example can be constructed by stipulating that the concrete physical implementation of measuring $\mathcal{A B}$ and the other joint quantities in Eq. (15), in the two-particle singlet state, be: "Measure $\sigma_{x}^{\mathrm{I}}$ and $-\sigma_{x}^{\mathrm{II}}$, call the results "spin of particle I along axis $\alpha$ and spin of particle II along axis $\beta$, respectively;" perform the same measurement of $\sigma_{x}^{\mathrm{I}}$ and $-\sigma_{x}^{\mathrm{II}}$ to obtain the spin values for the pairs of axes $\alpha^{\prime}, \beta$ and $\alpha, \beta^{\prime}$, and measure $\sigma_{x}^{\mathrm{I}}$ and $+\sigma_{x}^{\mathrm{II}}$ in the case of $\alpha^{\prime}, \beta^{\prime}$." Obviously, the correlation functions obtained in this way violate Cirel'son's inequality maximally (even though outcomes and probabilities at each wing are insensitive to what choice is made at the other wing). This is because the quantities defined in this way are not bona fide local physical quantities in the sense we have discussed. Indeed, according to this measurement protocol the measuring procedure for the spin of particle II along $\beta^{\prime}$ depends on whether spin along $\alpha$ or along $\alpha^{\prime}$ is measured on particle I.

So we have identified a first locality requirement: the operators that are used to represent physical quantities on the individual wings of the experiment should refer to the same physical devices and interactions, regardless of what goes on at the other side. It should be possible to measure these quantities on wing 1 and wing 2, respectively, together; therefore the operators representing them should be compatible (i.e., commuting or jointly measurable in the sense of the POVM scheme).

The second locality assumption to be considered is that no signals are transmitted: the possible outcomes and their probabilities on the two sides of the experiment are insensitive to what happens at the other wing. Usually, this is the only assumption that is explicitly discussed.

Within the orthodox treatment of measurements in quantum theory the compatibility assumption and the nosignaling assumption are equivalent: both lead to the requirement that the operators at one side commute with those at the other side. This commutativity is in turn sufficient to derive Cirel'son's inequality and its generalizations, including inequality (15). Within the framework of generalized measurements it is the no-signaling requirement that leads to commutativity; so here we have to invoke locality (in the sense of no signaling, or relativistic causality in the relativistic context) explicitly in order to derive (15); the requirement of joint measurability is not enough.

The two just-mentioned locality requirements together lead to a theoretical description with one-wing operators (referring to invariant measuring procedures) that commute with those at the other wing. This is sufficient for deriving the inequalities of Sec. IV. These inequalities can therefore be used in experimental tests of locality. If an inequality is violated in experiments, this indicates either the propagation of influences that change the outcomes and/or their probabilities (an application of this idea can be found in [9]), or it indicates noninvariance of the measured quantity (see above for an example of the latter possibility) [11].

\section{AN ARGUMENT BY POPESCU AND ROHRLICH}

The conclusion of the preceding section is that quantum mechanical locality (no-signaling) is responsible for the existence of the upper bound of Cirel'son's inequality. This conclusion might seem in conflict with an argument by Popescu and Rohrlich [3]. These authors argue that the impossibility of signaling does not limit the sum of the correlations occurring in Cirel'son's inequality to $2 \sqrt{2}$. Their counterexample is an EPR situation in which spin measurements are performed on the two wings. For the outcomes of these measurements a particular probability distribution is postulated, as follows. The two possible outcomes are taken to be +1 and -1 along any axis, and both of these possibilities are postulated to have a probability of $1 / 2$, independently of the 
measurement performed at the other wing. So the measured outcomes and their probabilities do not give any information about choices made at the other side; it is impossible to signal, or, as Popescu and Rohrlich put it, relativistic causality is satisfied.

Further, for any pair of axes, the combinations of outcomes $+1,+1$ and $-1,-1$ are assumed to be equally probable, and the same applies to the combinations $+1,-1$ and $-1,+1$. Finally, the correlation function (a "superquantum" correlation function) is stipulated to have a form like the following:

$$
E(\theta)= \begin{cases}+1 & \text { for } 0 \leqslant \theta \leqslant \pi / 4 \\ 2-4 \theta / \pi & \text { for } \pi / 4 \leqslant \theta \leqslant 3 \pi / 4 \\ -1 & \text { for } 3 \pi / 4 \leqslant \theta \leqslant \pi\end{cases}
$$

This is equivalent to assuming that the probability $p_{++}(\theta)$ of the pair of outcomes $+1,-1$ is given by

$$
p_{++}(\theta)=\frac{E(\theta)+1}{4} .
$$

In these formulas $\theta$ is the angle between the axes on the left and right, respectively, along which the spin measurements are made.

Now consider four axes $\alpha^{\prime}, \beta, \alpha, \beta^{\prime}$ separated by successive angles of $\pi / 4$ and lying in one plane. We find that

$$
E(\alpha, \beta)+E\left(\alpha^{\prime}, \beta\right)+E\left(\alpha, \beta^{\prime}\right)-E\left(\alpha^{\prime}, \beta^{\prime}\right)=4 .
$$

Cirel'son's inequality can therefore be violated, even to the maximum extent logically possible, by a correlation function that respects invariance of one-wing outcomes and probabilities, and thus the impossibility of signaling.

Clearly, therefore, the requirement that outcomes and probabilities are invariant is by itself insufficient to derive Cirel'son's inequality and its generalizations. Nevertheless, we have demonstrated above that Cirel'son's inequality can be derived from that locality assumption within the theoretical framework of quantum mechanics. It is only when the nosignaling requirement is implemented within a well-

[1] B. S. Cirel'son, Lett. Math. Phys. 4, 93 (1980).

[2] L. J. Landau, Phys. Lett. A 120, 52 (1987).

[3] S. Popescu and D. Rohrlich, Found. Phys. 24, 379 (1994); e-print quant-ph/9508009; e-print quant-ph/9605004; e-print quant-ph/9709026.

[4] G. Ludwig, Einführung in die Grundlagen der Theoretischen Physik (Vieweg, Braunschweig, 1976), Vol. 3.

[5] E. B. Davies, Quantum Theory of Open Systems (Academic Press, New York, 1976).

[6] J. Uffink, Int. J. Theor. Phys. 33, 199 (1994).

[7] P. Busch and J. Singh, Phys. Lett. A 249, 10 (1998).

[8] J. Uffink, Phys. Rev. Lett. 88, 230406 (2002).

[9] D. Beckman, D. Gottesman, M. A. Nielsen, and J. Preskill, defined theoretical framework, equipped with prescriptions for how to represent observables (corresponding to characteristic measuring procedures), that it gets enough bite to make the derivation of Bell-type inequalities possible. Indeed, we have seen that within the framework of theories that operate with a phase space on which physical quantities are represented by (stochastic) functions, the original Bell inequalities can be obtained, whereas within the Hilbert space formalism of quantum theory inequality (15) results. In both cases the correlation function postulated by Popescu and Rohrlich cannot arise in a local way (it can be produced by non-local means as was illustrated earlier in this section).

The fact that within the framework of quantum theory the correlation function (16) cannot be produced in a local way [because it violates Eq. (15)] does not mean, of course, that there cannot exist other theoretical frameworks in which "superquantum" correlation functions could arise in a local way; frameworks that use neither functions on a state space nor the Hilbert space operator formalism. It is difficult to say anything definite about such hypothetical theoretical frameworks. Popescu's and Rohrlich's argument does not use any assumption about how the observables are represented mathematically, and therefore does not provide a sufficient basis for a discussion of locality and causality (in fact, we already observed that their probability distribution could be produced by non-local mechanisms, which implies that invariance of outcomes and probabilities is a necessary but not a sufficient condition for causality).

Summing up, Popescu and Rohrlich are right in their claim that Bell-type inequalities do not follow from the nosignaling requirement alone. But this does not answer the question of whether locality, in the sense of the no-signaling requirement, may lead to such inequalities in the context of specific theories. It turns out that if locality is fleshed out within classical theories, this leads to Bell's inequality; if it is fleshed out within the framework of quantum theory this leads to inequality (15).

\section{ACKNOWLEDGMENT}

I thank Jos Uffink for helpful remarks.
Phys. Rev. A 64, 052309 (2001).

[10] A. Cabello, Phys. Rev. Lett. 88, 060403 (2002); e-print quant-ph/0205183.

[11] This conclusion assumes that the experiments in question pertain to systems prepared in a quantum state $\rho$, so that the expectation value of $A$ is given by $\langle A\rangle=\operatorname{Tr}\{\rho A\}$. If one considers ensembles that cannot be represented by a quantum state, for example if one calculates averages in post-selected ensembles, there is in general no reason to expect that Cirel'son's inequality will apply. Cabello has recently given explicit examples [10] of situations in which Cirel'son's inequality is violated because post-selected ensembles are used for the calculation of average values. 